Z. klin. Chem. u. klin. Biochem.

9. Jg., S. 126 - 129, März 1971

\title{
Untersuchungen zum katabolen Mucopolysaccharid-Proteinstoffwechsel der Rattenleber nach Gabe von Äthanol
}

\author{
Von D. Platt, U. Stein und H. Hetssmeyer \\ Mediziniscbe Kliniken und Polikliniken der Justus Liebig-Universität Gießen (Direktorium: Prof. Dr. H.J. Dengler, \\ Prof. Dr. H. G. Lascb) \\ und Medizinische Klinik der Universität Freiburg (Direktorium: Prof. Dr. G. W. Löbr, Prof. Dr. W. Gerok)
}

(Eingegangen ami 16. November 1970)

In der rorliegenden Arbeit wird an 20 männlichen Wistar-Ratten der Einfluß von Alkohol (1 g Äthanol-16proz. ( $/ / v)$ - pro kg Körpergewicht i. p. für 14 Tage) auf das Aktivitätsverhalten der folgenden lysosomalen Enzyme untersucht: Hyaluronidase, $\beta$-Glucuronidase, $\beta$-Acetylglucosaminidase, Kathepsin und saure Carboxypeptidase. Während die $\beta$-Glucuronidase, $\beta$-Acetylglucosaminidase und saure Carboxypeptidase in der Lysosomenfraktion einen signifikanten Aktivitätsabfall aufweisen, fallen die Aktivitäten der Hyaluronidase und $\beta$-Glucuronidase im Lysosomenfraktions-Überstand signifikant ab. Die Proteinkonzentration fällt in der Lysosomenfraktion signifikant ab und bleibt im Lysosomenfraktions-Überstand konstant.

\section{The effect of ethanol on the catabolism of mucopolysaccharide-proteins in rat liver}

Ethanol $(1 \mathrm{~g}$ ethanol - 16\% (v/v) - per kg body weight i. p. for 14 days) was administered to 20 male wistar rats and the activities of the following lysosomal enzymes were studied: hyaluronidase, $\beta$-glucuronidase, $\beta$-acetylglucosaminidase, cathepsin and acid carboxypeptidase. In the lysosome fraction, there was a significant decrease in the activities of $\beta$-glucuronidase, $\beta$-acetylglucosaminidase and acid carboxypeptidase, while the activities of hyaluronidase and $\beta$-glucuronidase decreased significantly in the supernatant of the lysosome fraction. The protein concentration decreased significantly in the lysosome fraction and remained constant in the lysosome fraction supernatant.

Akute und chronische Äthanolzufuhr führt sowohl beim Menschen als auch in tierexperimentellen Untersuchungen zu morphologischen und biochemischen Veränderungen (1-5). Elektronenoptisch findet man schwerste Veränderungen an Mitochondrien und Lysosomen. Biochemisch zeigen sich Aktivitätsänderungen der Aspartat-Transaminase, Alanin-Transaminase, Sorbitdehydrogenase, Alkoholdehydrogenase u. a. $(3,4)$. Bei chronischem Alkoholgenuß wird bei vielen Patienten das Stadium der Fettleber durch die Fettleberhepatitis beendet (5). In einem gewissen Prozentsatz $(5-60 \%)$ entwickelt sich eine Leberzirthose (6). Als biochemisches Äquivalent der Zirrhose findet man eine Vermehrung des Skleroproteins Kollagen. In der menschlichen Leber steigt der Kollagengehalt im Stadium der Cirrhose um das 4-8fache gegenüber gesunden Lebern an (9). Untersuchungen von Mathews (12) sprechen dafür, daß möglicherweise Mucopolysaccharide an der Kontrolle der Kollagen-Synthese in Form eines Informationsschlüssels - beteiligt sind. In diesem Zusammenhang erschien es sinnvoll, Untersuchungen zum Mucopolysaccharidstoffwechsel bei der Äthanol-geschädigten Leber durchzuführen. BECKER (10) konnte durch Konzentrationsmessungen von Glucosamin, Galaktosamin und Uronsäure in cirrhotisch umgewandelten menschlichen Lebern zeigen, $\mathrm{da} \beta$ es zu Verschiebungen in den Konzentrationsverhältnissen der einzelnen Bausteine kommt. In der vorliegenden
Arbeit sollte nun der Frage nachgegangen werden, inwieweit es nach Gabe von Äthanol an Ratten zu Aktivitätsänderungen der am Abbau der Mucopolysaccharid-Proteinkomplexe beteiligten Enzyme: Hyaluronidase (EC 3.2.1.35), $\beta$-Glucuronidase (EC 3.2.1.31), $\beta$-Acetylglucosaminidase (EC 3.2.1.30), Kathepsin D (EC 3.4.4.23) und „saure" Carboxypeptidase (EC 3.4.2.) $)^{1}$ ) kommt.

\section{Methodik}

Für die experimentellen Untersuchungen wurden 20 männliche Wistar-Ratten mit einem durchschnittlichen Gewicht von $200 \mathrm{~g}$ verwendet. Die Tiere wurden bei einer Raumtemperatur von $23^{\circ}$ bei Trockenfutter der Firma Altromin und Wasser ad libitum gehalten. Den Versuchsserien liefen entsprechende Kontrollserien von 10 Tieren parallel. Die Tiere exhielten täglich $1 \mathrm{~g}$ Âthanol (16 Vol\%)/kg KG i. p. für 14 Tage. 24 Stdn. nach der letzten Gabe wurden die Ratten durch Dekapitation getötet und entblutet. Die Leber wurde sofort entnommen, gewogen und bei $4^{\circ}$ im Kühllabor für die histologischen und biochemischen Untersuchungen präpariert. Für die lichtmikroskopischen Untersuchungen wurden $5 \mu \mathrm{m}$ dicke Paraffinschnitte angefertigt und folgende Färbeverfahren angewendet: Hämatoxylin-Eosin und Sudan-Färbung sowie die PAS-Reaktion. Zur elektronenoptischen Darstellung wurden die Gewebsproben 2 Stdn. in 5proz. Glutaraldehyd mit $0,1 \mathrm{M}$ Cacodylat-Puffer, $\mathrm{pH} \quad 7,2$, vorfixiert und anschließend in 1 proz. isotonischer gepufferter $\mathrm{OsO}_{4}$-Lösung,

1) In der Enzym Nomenclature, Elsevier Publ. Comp. 2nd edition, Amsterdam 1965 enthaltenen Liste ist der systematische Name nicht verzeichnet. 
pH 7,2, nachfixiert. Die Einbettung erfolgte in Vestopal. Nachkontrastierung der Dünnschnitte mit Uranylacetat und Bleiacetat. Elektronenmikroskop EM 9, Zeiss. Zur Bestimmung der Enzymaktivitäten wurde das Lebergewebe zunächst in der 3fachen Menge einer 0,25 $\mathrm{M}$ Saccharose-Lösung, $\mathrm{pH}$ 7,2, in einem PotterElvehjem-Homogenisator homogenisiert und nach der Methode von DE DUVE (13) fraktioniert zentrifugiert. Das Sediment wurde nach 3 Einfrier-Auftauschritten zur Bestimmung der gebundenen lysosomalen Aktivität, der Lysosomensediment-Uberstand zur Bestimmung der freien lysosomalen Aktivität eingesetzt. Als Bezugssystem für die Enzymaktivitäten diente die Proteinkonzentration in den einzelnen Präparationen. Die Methodik der Enzymaktivitätsmessungen geht aus früheren Untersuchungen hervor (14).

\section{Ergebnisse}

Aus den Ergebnissen geht hervor, daß die am Abbau des Mucopolysaccharid-Proteinkomplexes beteiligten Glycosamino-Glycanohydrolasen und Peptidyl-Peptidhydrolasen ein unterschiedliches Aktivitätsverhalten nach Äthanolgabe aufweisen. So zeigt die $\beta$-Glucuronidase und $\beta$-Acetylglucosaminidase im Lysosomensediment einen signifikanten Aktivitätsabfall, während die freie lysosomale Aktivität der Hyaluronidase und $\beta$ Glucuronidase signifikant abfällt, die der $\beta$-Acetylglucosaminidase konstant bleibt (Abb. 1-3 und Tab. 1). Das Aktivitätsverhalten der sauren Carboxypeptidase und des bei $\mathrm{pH}$ 3,2 optimal Hämoglobin-spaltenden Kathepsin geht aus den Abbildungen 4 und 5 sowie aus Tabelle 2 hervor. So sieht man, daß lediglich die Aktivität der sauren Carboxypeptidase im Lysosomensediment einen signifikanten Aktivitätsabfall erkennen läßt. Die Proteinkonzentration fällt im Lysosomensediment signifikant $a b$, bleibt im LysosomensedimentÚberstand dagegen konstant (Abb. 6 und Tab. 1).

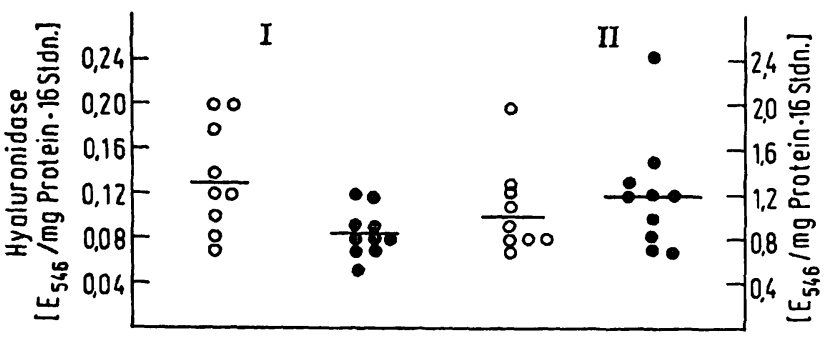

Abb. 1

Anderungen der Hyaluronidase-Aktivitäten der Rattenleber nach Gabe von Alkohol (•). Kontrolitiere (o)

$I$ = Lysosomensediment-Überstand, II = Lysosomensediment

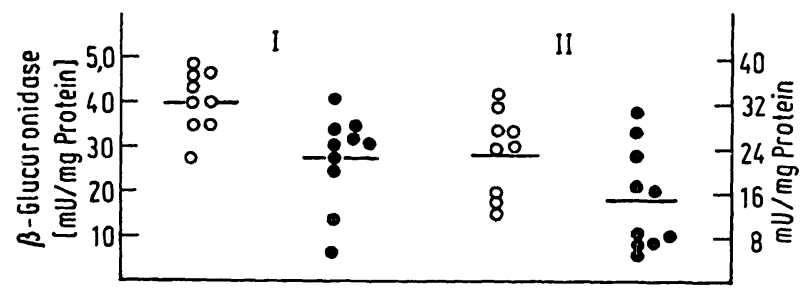

Abb. 2

Anderungen der $\beta$-Glucuronidase-Aktivitäten der Rattenleber nach Gabe von Alkohol (•). Kontrolltiere (o)

I = Lysosomensediment-Uberstand, II = Lysosomensediment

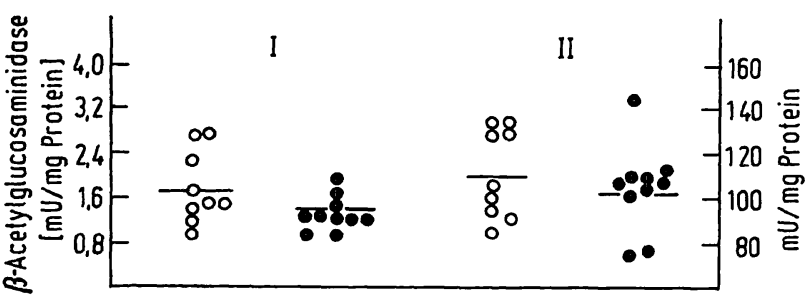

Abb. 3

Änderungen der $\beta$-Acetylglucosaminidase-Aktivitäten der Rattenleber nach Gabe von Alkohol (•). Kontrolltiere (0)

$\mathrm{I}=$ Lysosomensediment-Überstand, II = Lysosomensediment

Tab. 1

Statistische Daten zu den Abbildungen 1, 2, 3 und 6

\begin{tabular}{|c|c|c|c|c|c|c|c|c|}
\hline \multirow[t]{2}{*}{ Enzyme/Protein } & \multicolumn{3}{|c|}{ Kontrolltiere (a) } & \multicolumn{3}{|c|}{ Versuchstiere (b) } & \multicolumn{2}{|c|}{$a / b$} \\
\hline & $\mathbf{n}$ & $\overline{\mathbf{x}}$ & s & $\mathrm{n}$ & $\overline{\mathbf{x}}$ & s & $t$ & $\mathrm{p}$ \\
\hline \multirow{2}{*}{$\begin{array}{l}\text { Lysosomensediment } \\
\text { Hyaluronidase }\left(\mathrm{E}_{\mathrm{s}, \mathrm{c}} \mathrm{nm} / \mathrm{mg} \text { Protein } \cdot 16 \mathrm{Stdn} .\right) \\
\text { Lysosomensedimentüberstand }\end{array}$} & 10 & 1,08 & 0,39 & 10 & 1,21 & 0,55 & 0,61 & $<0,30$ \\
\hline & 10 & 0,13 & 0,05 & 10 & 0,085 & 0,02 & 2,75 & $<0,005$ \\
\hline \multirow{2}{*}{$\begin{array}{l}\text { Lysosomensediment } \\
\beta \text {-Glucuronidase (mU/mg Protein) } \\
\text { Lysosomensedimentüberstand }\end{array}$} & 10 & 23,43 & 7,76 & 10 & 15,10 & 9,67 & 1,92 & $<0,05$ \\
\hline & 10 & 4,00 & 0,70 & 10 & 2,80 & 1,00 & 9,62 & $<0,0005$ \\
\hline \multirow{2}{*}{$\begin{array}{l}\text { Lysosomensediment } \\
\beta \text {-Acetylglucosaminidase (mU/mg Protein) } \\
\text { Lysosomensedimentüberstand }\end{array}$} & 10 & 112,40 & 20,82 & 10 & 105,30 & 19,37 & 1,80 & $<0,05$ \\
\hline & 10 & 1,77 & 0,64 & 10 & 1,37 & 0,28 & 0,77 & $<0,25$ \\
\hline \multirow{2}{*}{$\begin{array}{l}\text { Lysosomensediment } \\
\text { Protein ( } \mathrm{mg} / \mathrm{ml}) \\
\text { Lysosomensedimentüberstand }\end{array}$} & 10 & 5,90 & 0,87 & 10 & 4,62 & 0,77 & 3,4 & $<0,0025$ \\
\hline & 10 & 13,50 & 0,79 & 10 & 14,30 & 1,37 & 1,53 & $<0,10$ \\
\hline
\end{tabular}

Tab. 2

Statistische Daten zu den Abbildungen 4 und 5

\begin{tabular}{|c|c|c|c|c|c|c|c|c|}
\hline \multirow{2}{*}{ Enzyme } & \multicolumn{3}{|c|}{ Kontrolltiere (a) } & \multicolumn{3}{|c|}{ Versuchstiere (b) } & \multicolumn{2}{|c|}{$a / b$} \\
\hline & $n$ & $\overline{\mathbf{x}}$ & $\mathrm{s}$ & $\mathbf{n}$ & $\bar{x}$ & $\mathbf{s}$ & $\mathbf{t}$ & p \\
\hline \multirow{2}{*}{$\begin{array}{l}\text { Lysosomensediment } \\
\text { Kathepsin D (mU/mg Protein) } \\
\text { Lysosomensedimentüberstand }\end{array}$} & 10 & 3,74 & 1,29 & 10 & 3,88 & 1,27 & 0,23 & $<0,45$ \\
\hline & 10 & 0,63 & 0,12 & 10 & 0,52 & 0,18 & 1,55 & $<0,10$ \\
\hline \multirow{2}{*}{$\begin{array}{l}\text { Lysosomensediment } \\
\text { Saure Carboxypeptidase (mU/mg Protein) } \\
\text { Lysosomensedimentüberstand }\end{array}$} & 10 & 13,40 & 4,40 & 10 & 13,10 & 4,20 & 0,14 & $<0,45$ \\
\hline & 10 & 0,49 & 0,10 & 10 & 0,37 & 0,08 & 2,88 & $<0,01$ \\
\hline
\end{tabular}




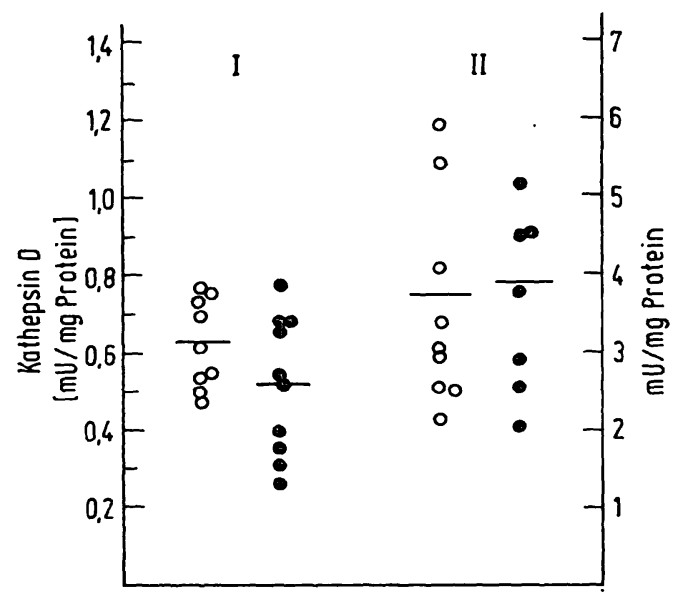

Abb. 4

Anderungen der Kathepsin D-Aktivitäten der Rattenleber nach Gabe von Alkohol (•). Kontrolltiere (o)

$I$ = Lysosomensediment-Überstand, II = Lysosomensediment

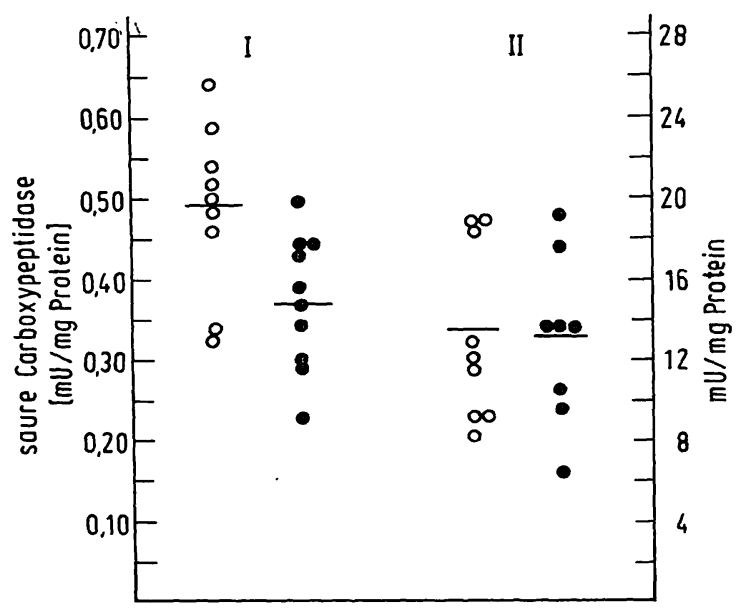

Abb. 5

Änderung der sauren Carboxypeptidase-Aktivitäten der Rattenleber nach Gabe von Alkohol (•). Kontrolltiere (o)

I = Lysosomensediment-Oberstand, II = Lysosomensediment

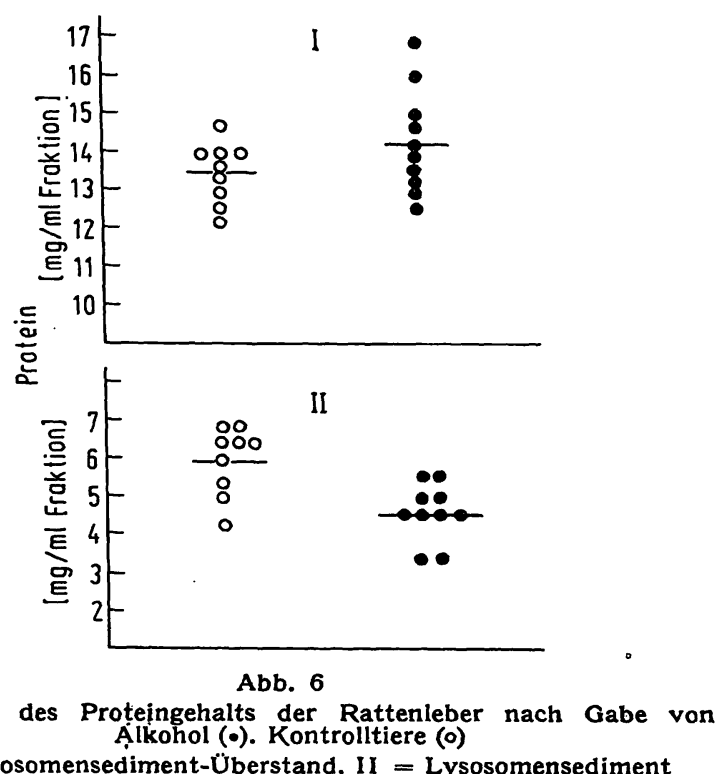

Anderungen des Proteingehalts der Rattenleber nach Gabe von
Ạlkohol (०). Kontrolltiere (o) I = Lysosomensediment-Überstand, II = Lysosomensediment

\section{Diskussion}

BECKER (10) konnte zeigen, daß es in cirrhotisch umgewandelten menschlichen Lebern zu einer Abnahme des Glucosamin-Uroṇsäure-Quotienten kommt, ein Befund, der dafür spricht, daß bei der Proliferation von Bindegewebe in der Grundsub́stanz in erster Linie saure Glycosaminoglycane synthetisiert werden, in geringerem Maße neutrale Mucopolysaccharide (10). Gleichzeitig geht aus den Untersuchungen hervor, daß in der cirrhotischen Leber besonders Dermatansulfat gebildet werden dürfte. Mit der Einführung radioaktiv markierter Substanzen konnten zahlreiche Syntheseschritte der Mucopolysaccharide aufgeklärt werden. BECKER untersuchte die Inkorporationsraten von ${ }^{35} \mathrm{SO}_{4}$ in die sauren Mucopolysaccharide der alkoholvorgeschädigten Leber der Ratte (11). Die Untersuchungsergebnisse zeigen, daß die Inkorporationsraten in die sauren Mucopolysaccharide der Leber bereits zu einem Zeitpunkt Veränderungen aufweisen, in dem morphologisch noch keine faßbaren Veränderungen sichtbar sind. Im Langzeitversuch fand BECKER, daß der ${ }^{35} \mathrm{SO}_{4}$ Einbau in die Leber bei chronischer Alkoholintoxikation erhöht ist. TAkeuchr und Prockop (15) fanden eine Zunahme der Peptidyl-Prolin-Hydroxlyase-Aktivität bei der Fibrose. Unsere Untersuchungen zeigen, $\mathrm{da} ß$ die am Abbau des Mucopolysaccharid-Proteinkomplexes beteiligten Enzyme nach Alkoholgabe signifikante Aktivitätsänderungen aufweisen. Vergleicht man die gebundenen Aktivitäten der GlycosaminoGlycanohydrolasen mit denen der Peptidyl-Peptidhydrolasen so sieht man, daß beide Enzymgruppen ein unterschiedliches Verhalten aufweisen. Während die Aktivitäten der Peptidyl-Peptidhydrolasen gleichbleiben, fallen die Aktivitäten $\operatorname{der} \beta$-Glucuronidase und $\beta$-Acetylglucosaminidase signifikant ab. Für diesen Aktivitätsabfall sprechen die ultrastrukturellen Veränderungen (PLATT und SCHNORR, 1970), aus denen hervorgeht, daß neben den schweren mitochondrialen Veränderungen die Lysosomen eine intakte Membran vermissen lassen. $\mathrm{Da}$ jedoch gleichzeitig im Lysosomensediment-Überstand ein Aktivitätsabfall der Hyaluronidase, $\beta$-Glucuronidase und sauren Caroboxypeptidase nachweisbar ist, scheint es wahrscheinlich, daß die Enzyme inzwischen den Zellularraum verlassen haben und möglicherweise in die Blutbahn gelangt sind. Aktivitätsmessungen im Blut haben wir in der vorliegenden Arbeit richt durchgeführt. Jedoch könnten die Arbeiten von voN OLDERShausien und SchweIger für einen Aktivitätsverlust der Enzyme in das Blut sprechen. Diese Autoren konnten nämlich zeigen, daß es nach Gabe von Alkohol zu einem Aktivitätsanstieg der Aspartat- und Alanintransaminase sowie Sorbitdehydrogenase im Serum kommt. Eine gesteigerte Inkorporationsrate von ${ }^{35} \mathrm{SO}_{4}$ (11) bei der chronischen Alkoholintoxikation und die in den vorliegenden Untersuchungen gezeigten Aktivitätsabnahmen der katabolen Enzyme sprechen für eine Verschiebung des Gleichgewichtes Synthese - Abbau zugunsten der Synthese. In Übereinstimmung damit stehen die vermehrten Ablagerungen von Glycosaminoglycanen und Skleroproteiden im chronischen Stadium der Alkoholschädigung $(16,17)$. 


\section{Literatur}

1. Bode, CH., H. Goebell und M. Stähler, Zschr. exper. Med. 152, 111 (1970). - 2. Rubin, E. und CH. S. Lieber, Gastroenterology, Baltimore 52, 1 (1967). - 3. von Oldershausen, H. F. und M. Schwerger, Verh. Dtsch. Ges. inn. Med. 70, 645 (1964). 4. Platt, D. und B. Schnorr, Arzneimittel Forsch., Aulendorf, im Druck. - 5. Becketr, A. G., A. V. Livingstone und K. R. HrLl, Brit. Med. J. II, 1113 (1961). - 6. Bötrner, H., Med. Welt, 52, 2857 (1968). - 7. MaliorX, F. B., Bull. Johns Hopkins Hosp., 22, 69 (1911). - 8. ThaleR, H. Gastroenterologia (Basel), Suppl. ad. Vol. 95, 246 (1961). - 9. Huttner, F., E. Rubin, E. J. Singer und A. Popper, Proc. Soc. Exper. Biol. Med., N. Y. 102, 534 (1959). - 10. BeCKER, K., Zschr. exper. Med. 151, 1
(1961). - 11. BeCkER, K., Internat. Symposium „Alkohol und Leber", Schattauer Verlag, im Druck. - 12. Mathews, M. B., Biochem. J. 96, 710 (1965). - 13. De Duve, C., R. Wattiaux und M. Wiвo, Biochem. Pharmacol. 9, 97 (1962). - 14. Platt, D. und U. Strein, diese Z. 7, 374 (1969). - 15. TAkEUCHI, T. und D. J. Prockop, Gastroenterology, Baltimore, 56, 744 (1969). 16. Hutterer, F. und P. Bacchin, Federation Proc. 27, 412 (1968). - 17. Yamauchi, F., M. Kosakai und Z. Yosizawa, Biochem. Biophys. Res. Comm. 33, 721 (1968). - 18. Hutterer, F., M. Eisenstadt und E. Rubin, Experientia, Basel, 26, 244 (1970).
Priv. Doz. Dr. D. Platt

Medizinische Univ. Klinik

63 Gießen

Klinikstr. $32 b$

Dr. U. Stein

und Dr. H. Heißmeyer

Medizinische Univ. Klinik

78 Frciburg 